\title{
The path to herd immunity: is mandatory vaccination the answer?
}

\author{
Steven S. Cheng ${ }^{1, *}$ and Bianca A. Lepe ${ }^{2}$ \\ Edited by Sumedha Sachar and Friederike M. C. Benning
}

\section{HIGHLIGHTS}

- Vaccines have significantly reduced infectious disease burden in society, saving numerous lives and improving people's health.

- Aiming to increase vaccination rates among the population, some policymakers have considered instituting vaccine mandates.

- Vaccine mandates should be crafted carefully to ensure that exemptions are given when necessary, that they do not exacerbate societal inequities, and that they do not provoke societal backlash.

Vaccination is a public health measure that is routinely performed to reduce the risk of contracting a particular infectious disease. Taking advantage of the body's immune system, vaccination can confer immunity against disease-causing pathogens. Though vaccination generally is not able to yield absolute immunity, the procedure has nonetheless improved public health and reduced infectious disease mortality globally, saving millions of lives each year [1]. If a high enough proportion of a population obtains immunity to a particular pathogen such that the spread of the associated disease has slowed and members of the population without immunity are also protected, then herd immunity has been achieved. Consequently, there have been a number of debates about using policymaking tools to achieve this high population vaccination rate, including policies of mandatory vaccination. Here, we discuss the scientific background of vaccination, present frameworks for understanding the arguments for and against mandatory vaccination policies, and highlight data and a case study in support of these arguments within the context of the United States. We hope to better inform the policymaking community of the factors that must be weighed when considering a mandatory vaccination policy.

\section{Scientific background of vaccination}

How do vaccines work?

\footnotetext{
${ }^{1}$ Department of Chemistry and Chemical Biology, Harvard University, Cambridge, MA

${ }^{2}$ Department of Biological Engineering, Massachusetts Institute of Technology, Cambridge, MA

*Email: scheng1@g.harvard.edu
}

The authors declare no conflict of interest.

(C) 2021 The Author(s) nfectious diseases are caused by pathogens such as bacteria, viruses, fungi, and parasites that invade the human body. Typically, vaccines consist of live attenuated or dead pathogens, no longer able to cause disease, along with an adjuvant. The body's immune system recognizes the former as a foreign substance and begins to mount an immune response directed against the pathogen through the production of proteins that protect cells called neutralizing antibodies. Adjuvants are substances used in vaccines to promote a robust immune response. By training the immune system to recognize and neutralize a particular pathogen, vaccination substantially decreases a person's risk of becoming severely infected and/or ill upon exposure to that pathogen (Fig. 1) [2]. Recent advances in biotechnology have led to other types of effective and easy-to-produce vaccines, e.g., messenger RNA vaccines and purified antigen vaccines.

The efficacy of a vaccine is calculated from the difference between the number of infections in a vaccinated group and a control or placebo group (which is not given the vaccine) in a randomized clinical trial. Vaccine effectiveness is similarly calculated based on how well the vaccine performs in the general population. Different vaccines often have different efficacies depending on what disease they are aimed against, what delivery technology they use, and other patient-specific factors such as age [3]. For example, some vaccines are highly effective; the Centers for Disease Control and Prevention (CDC) reports that the polio vaccine is $99 \%$ to $100 \%$ effective with three doses and the measles, mumps, and rubella (MMR) vaccine is $97 \%$ effective with two doses $[4,5]$. For measles, an estimated 23 million lives have been saved since 2000 due to vaccines and the World Health Organization (WHO) annual reporting illustrates the drop of measles cases corresponding to the rise of vaccine coverage (Fig. 2) [6]. Recently developed messenger RNA-based vaccines by Pfizer/BioNTech and Moderna achieved roughly $95 \%$ efficacy in protection against COVID-19 


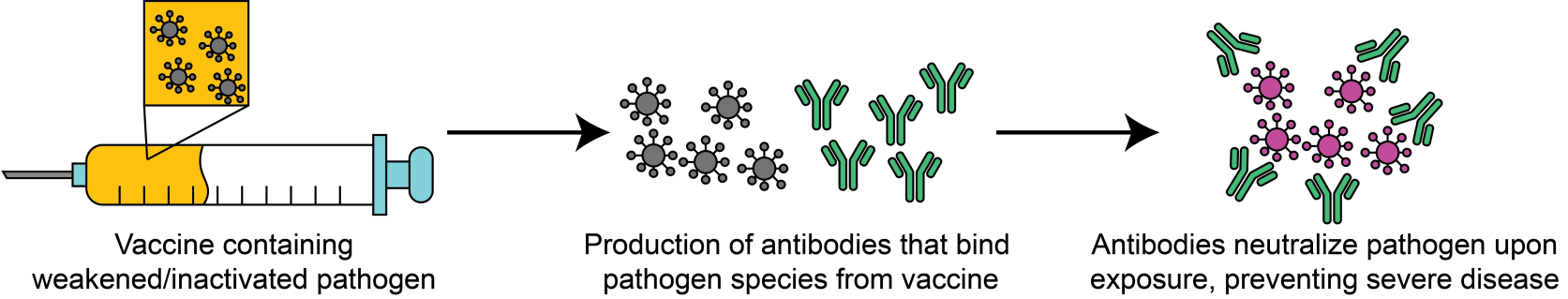

Figure 1: Schematic illustrating how live-attenuated/inactivated vaccines lead to immunity. In response to a vaccine, the body's immune system produces antibodies against specific pathogens which neutralize them the next time a person is exposed to the same pathogen, preventing severe disease.

and have been able to protect many lives $[7,8]$. On the other hand, the influenza vaccine is updated annually to account for changing influenza strains and has varying effectiveness. In years when the vaccine virus is matched with the circulating virus, the vaccine effectiveness is in the range of $40 \%-60 \%$, while in years when there are vaccine mismatches, the effectiveness can be as low as $13 \%$ as seen in the $2014-2015$ season [9].

What is herd immunity? Once a sufficient proportion of the population is immune to a pathogen, the population is said to have achieved herd immunity. When most of the population is susceptible to infection, then disease can rapidly spread between individuals (Fig. 3A). In herd immunity, the pathogen cannot efficiently spread between individuals as many are already immune, slowing the rate of infection and protecting those who do not have immunity (Fig. $3 B$ ). The level of immunity required to achieve herd immunity is mathematically defined based on $R_{0}$, which represents the average number of new infections that will arise from a single infected individual. Specifically, the herd immunity threshold is defined as $1-1 / R_{0}$ [10]. As $R_{0}$ is a mathematical abstraction, estimates of $R_{0}$ for any particular disease often vary greatly and can change depending on environmental conditions. In developing pandemic planning scenarios, the CDC estimates for the $R_{0}$ value of COVID-19 range from 2.0 to 4.0 , corresponding to herd immunity thresholds of $50 \%$ to $75 \%$ of the population [11]. The threshold proportion of the population that must be vaccinated to achieve herd immunity also may vary as individuals may become immune to a pathogen by natural exposure rather than through vaccination. As different pathogens spread at different rates, leading to different $R_{0}$ values, there are different thresholds for achieving herd immunity for different pathogens. Therefore, the relationship between vaccination initiatives and achieving herd immunity is complex and constantly changing.

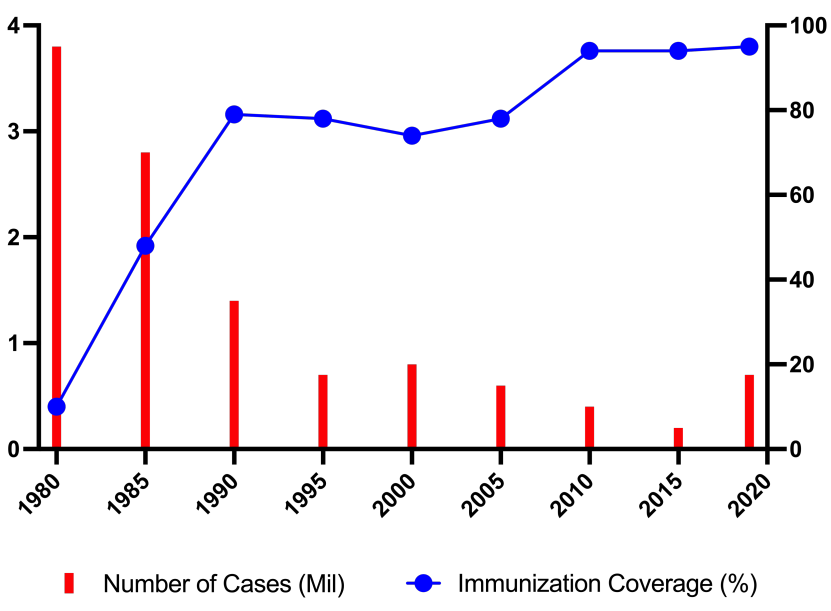

Figure 2: Number of measles cases (millions) versus immunization coverage percentage, globally from years 1980-2019. Note that as immunization coverage increases, disease cases decrease. Adapted from WHO [12].

Why are some people hesitant to be vaccinated? One major challenge in achieving high vaccination rates is countering disinformation and misinformation regarding vaccine safety. There have been numerous controversies regarding the safety of vaccines, most notably around a purported relationship between the MMR vaccine and autism, and the potential for vaccine-induced Guillain-Barré syndrome (GBS), a disease in which the body's immune system mistakenly attacks the nervous system. Consequently, vaccine hesitancy is a major issue in many societies, including the U.S., making it essential to contextualize or debunk the supposed risks of vaccination. The report suggesting a link between the MMR vaccine and autism has been retracted due to ethical misconduct and financial conflicts of interest. The scientific inaccuracies and fraudulent research practices in this study have led to widespread rejection by the scientific community [13]. Also, numerous studies have since debunked this proposed association. Vaccine-induced GBS became a concern during the 1976 swine flu vaccination program in the U.S. as the vaccine appeared to increase the risk of developing GBS. This association has so far largely 
been limited to the 1976 swine flu vaccine. Moreover, influenza infection has been found to be a greater risk factor for GBS than influenza vaccination, making vaccination preferable over natural exposure [14]. Some religious groups also show hesitance towards certain vaccines for many reasons, which are not further elaborated on in this work [15]. Specifically in the case of COVID-19 vaccines, some vaccine hesitancy also arises from the novelty of the messenger RNA vaccines and the speed with which the vaccines were developed [16]. Numerous studies, however, have demonstrated that the vaccines are safe and effective $[7,8]$.

Vaccines are not innocuous; they often lead to side effects such as fever, fatigue, muscle pain, joint pain, and headache. However, these side effects are validation that the vaccine is eliciting an immune response, and they are minor relative to the risks of infection with the active pathogen. Conducting randomized clinical trials and presenting safety and efficacy results in a clear, transparent manner is key to building public faith in vaccination programs. It should be noted that this is not sufficient; effective communication and other strategies discussed later must complement transparent vaccine development to encourage vaccine uptake. There are cases in which vaccination may pose certain health risks, such as with immunocompromised patients, whose immune systems have impaired function. In these cases, caution must be taken to fully understand the risks in order to prevent undesirable events, for example, infection by a live attenuated vaccine. While vaccines are not entirely without side effects, the medical and scientific communities should emphasize that minor side effects are common in the general population and the benefits of vaccination greatly outweigh the risks [17].

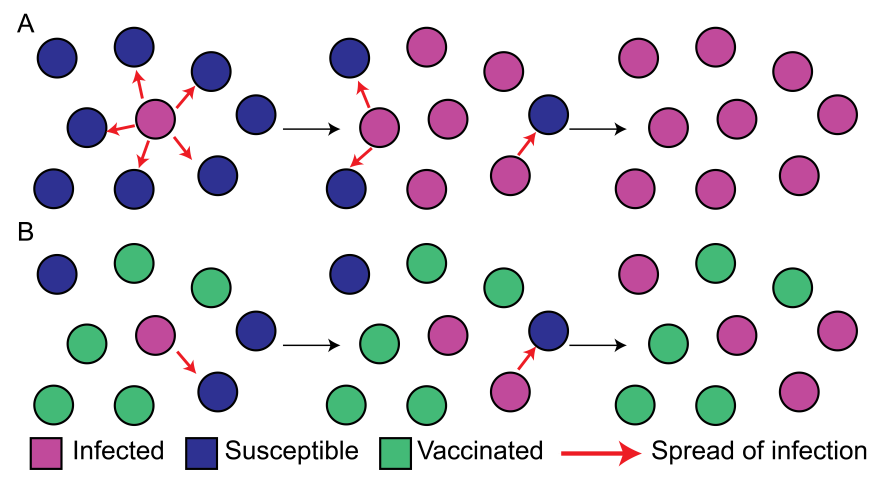

Figure 3: Illustration of how vaccination can lead to herd immunity and slow the spread of disease. (A) Spread of disease through a susceptible community. Everyone is exposed to disease and infected over time. (B) Spread of disease through a mostly vaccinated community. Immunity through vaccination prevents infections by blocking off paths for infection to spread.
There are numerous other factors that can impede vaccination campaigns including distribution to isolated communities, availability of medical staff, vaccine storage, and vaccine supply. As has been seen with COVID-19, these factors are especially important in the midst of an active pandemic when vaccine demand often exceeds vaccine supply [18]. Successful vaccination efforts depend on resolving each of these factors in addition to encouraging vaccine uptake.

\section{Ethical \& legal frameworks for analyzing mandatory vaccination}

As vaccinating a large enough proportion of the population to achieve herd immunity may encounter a number of potential roadblocks, some policymakers have proposed mandatory vaccination to accelerate the road to herd immunity. Before outlining data and case studies regarding vaccination policy, we discuss the ethical and legal frameworks for understanding the arguments around mandatory vaccination.

Is mandatory vaccination ethical? In a utilitarian analysis, the most beneficial policy option is one that maximizes societal benefits while minimizing societal costs [19]. Under this framework, some argue that vaccine mandates should be used to increase vaccination rates, as the lives saved and the public health benefits outweigh the loss of freedom for people to make independent decisions about vaccination [20]. In a contrary argument, some biomedical ethicists emphasize the importance of patient autonomy - patients should be able to make their own choices about whether or not to receive a particular healthcare intervention [19]. By definition, a vaccine mandate would infringe upon patient autonomy by financially or legally penalizing those who choose not to receive a vaccine, thus violating the clinician-patient relationship [21].

Is mandatory vaccination legal? In the U.S., vaccine mandates have long existed in various forms. At the beginning of the 20th century, the Commonwealth of Massachusetts allowed "the board of health of a city or town if, in its opinion, it is necessary for the public health or safety shall require and enforce the vaccination and revaccination of all the inhabitants thereof and shall provide them with the means of free vaccination" (1). Healthy adults refusing the vaccination would be fined. Henning Jacobson, who was fined under this policy, argued that this was a violation of the Fourteenth Amendment which states that "[n]o state shall make or enforce any law which shall abridge the privileges or immunities of citizens of the United States; nor shall any state deprive any person of life, liberty, or property, without due process of law; nor deny to any person within its jurisdiction the equal protection of the laws" (2). In Jacobson v. Massachusetts, the Supreme Court ruled that the vaccine mandate adopted by the City of Cambridge and authorized by the Commonwealth of Massachusetts was valid under the "police power of a State" to enact regulations that protect public health (3). This ruling was reaffirmed in Zucht $v$. King, in which the San Antonio school district was being sued for excluding unvaccinated students 
(4). Since then, school immunization mandates have existed across the U.S. and have been upheld numerous times in courts [22].

Presently, all fifty states have some form of vaccination requirement for enrolling in public schools. While all states offer medical exemptions to this requirement, there are differences in the non-medical exemptions (NMEs) offered. Specifically, 45 states and Washington D.C. grant religious exemptions to this requirement; 15 states also grant philosophical (personal, moral, or other) exemptions to this requirement (Fig. 4) [23]. Under the National Childhood Vaccine Injury Act, federal law requires that medical providers provide vaccine information statements prepared by the CDC to patients or their legal representative for a number of different vaccines (5). Parental consent for vaccination is generally required under state laws, though there are limited exceptions, such as for vaccination against sexually transmitted infections, through minor consent laws [24]. Some state legislatures have proposed bills allowing adolescents to consent to certain vaccines with the goal of achieving higher vaccination rates [24]. Policymakers considering vaccine mandates should keep these legal frameworks in mind when thinking about informed patient consent.

\section{Case study of widespread vaccination health outcomes}

When analyzing the advantages and disadvantages of mandatory vaccination, we can learn from past outbreak experiences. Of the several outbreaks that occurred in the U.S. in the last century, we highlight the case of measles. From this case study, we see that high vaccination rates can lead to significant disease reduction; however, undervaccination can lead to resurgences.

Vaccines helped reduce measles cases in the U.S., but undervaccination is leading to resurgences: Measles,

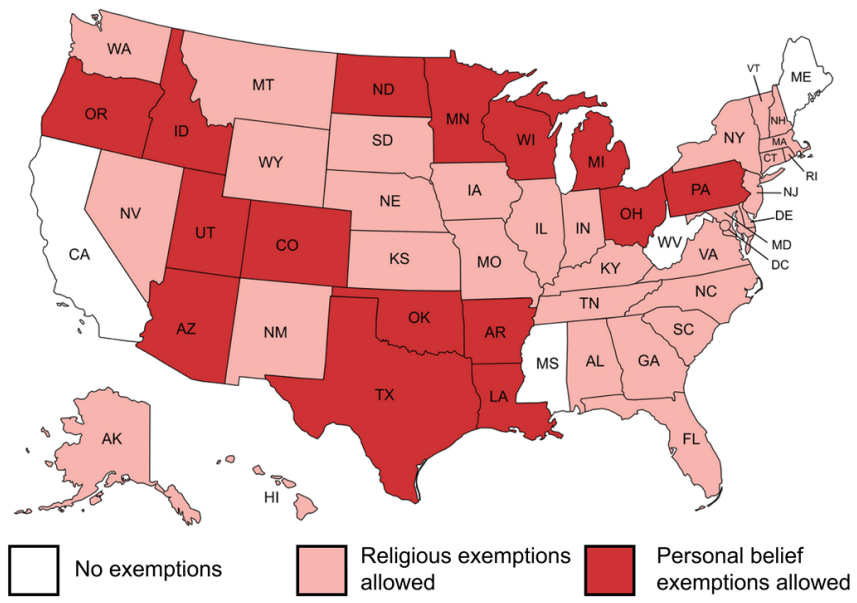

Figure 4: Map of non-medical exemptions from school immunization requirements by state in 2020 . White states do not allow any non-medical exemptions; pink states allow only religious exemptions; and red states allow exemptions due to any personal belief. Adapted from [23]. caused by the measles virus, is an illness characterized by a red flat rash preceded by runny nose, fever, coughs, and inflamed eyes. Before the introduction of a vaccine, it has been estimated that 3-4 million cases occurred per year with around 500 deaths reported annually [25]. A live attenuated, one-dose vaccine introduced in 1963 helped quickly reduce the reported incidence of the disease. Following some outbreaks among school children, a second dose was added to the schedule by the CDC's Advisory Committee on Immunization Practices in 1989 [26]. The U.S. has eliminated measles since 2000 according to $\mathrm{WHO}$.

A resurgence of measles cases occurred between 2018-2019, largely due to gaps in vaccination coverage. For example, in 2019, the U.S. had the largest case number (1282 cases) of measles since 2000 which caused states of emergency to be declared in some cities and states. Several causes are attributed to this rise, namely, clusters of undervaccinated individuals [27]. During this surge, a number of states tightened their vaccine exceptions in various ways. In New York City, Mayor de Blasio ordered mandatory vaccinations in specific neighborhoods [28]. In an alternative approach, Maine's state government eliminated the philosophical and religious exemptions for mandatory childhood vaccines (6). This decision was upheld in a 2020 referendum vote. Similarly, in the state of Washington, legislators put forth a law removing a parent's vaccination refusal due to personal/philosophical reasons, though they kept medical and religious exemptions (7). Although it originally applied to all mandatory childhood vaccines, it was modified to just the measles vaccine, given as the MMR vaccine, which protects against measles, mumps, and rubella, when passed. Overall, vaccination efforts within the U.S. significantly decreased the disease burden of measles.

\section{Important considerations for devising mandatory vaccination policies}

In this section, we further discuss some of the key points that must be taken into consideration when weighing policymaking for vaccine mandates including (1) exemptions to vaccine mandates, (2) the use of monetary incentives and/or disincentives to encourage vaccination, and (3) societal backlash to vaccine mandates and institutional distrust.

Who should be exempt from vaccines? Vaccine exemptions are granted for medical, religious and/or philosophical reasons. Medical exemptions exist because some individuals may have legitimate medical risks associated with vaccination such as severe allergic reactions or complications arising from a compromised immune system. Removing this type of exemption is considered an ethical violation. These exemptions are infrequent, so they do not significantly impede attainment of herd immunity through vaccination efforts. In the 2018-2019 school year, approximately $2.5 \%$ of kindergartners had an exemption for one or more required vaccines, of which only $0.3 \%$ were medical exemptions [29]. Religious exemptions are given to those who have religious objections to 
vaccination, while philosophical exemptions are given to those with objections based on personal or moral beliefs.

Policies around vaccination exemptions can be enacted to minimize outbreak risk by increasing vaccination coverage. Studies have found that states which have more lenient exemption policies tend to have lower childhood vaccination rates. For instance, from 2016-2018 on a state-aggregated level, NME rates were negatively associated with vaccination rates for three major childhood vaccine mandates: MMR, varicella, and diphtheria, tetanus, and acellular pertussis. In the analysis, spatial distributions of high rates of NMEs were found to be important considerations when assessing the risk of disease outbreaks [30]. Furthermore, states that enacted policy changes which eliminated either a subset, or all, NMEs corresponded to exemption rate reduction. One such state, California, eliminated NMEs in 2016 through their enactment of Senate Bill 277 following a measles outbreak in 2015 [31]. With its success in reducing vaccination exemption rates, California provides a sound legal basis for other states to also ban NMEs. However, a vaccination model that only has medical exemptions is still debated in terms of acceptability and enforceability. One alternative would be to eliminate NMEs specifically for diseases often transmitted in school settings in order to avoid bolstering anti-vaccine sentiments.

Another alternative to outright elimination proposes that NMEs should be rigorously reviewed and made administratively difficult to obtain. An argument against eliminating NMEs is that the so-called inconvenience approach is a more effective way to decrease exception rates as exemplified by the policies found in Michigan, which require education sessions and official state application forms for NMEs [32]. Similar trends favoring the inconvenience approach have been found in further studies, which show that increased procedural requirements to obtain NMEs for a mandated vaccination decreased the number of exemptions [33]. Furthermore, there is evidence that elimination of NMEs will not necessarily improve vaccination rates due to an increase in claims for medical exemptions and religious exemptions as well if only the philosophical exemptions are removed [34]. It is thought that a similar increase in medical exemptions could follow in response to increasing the difficulty of NME obtainment. Nevertheless, eliminating NMEs or making them harder to obtain are disputed in terms of preferability from an ethical perspective.

Monetary incentives \& disincentives: In mandatory vaccination policies, monetary measures are often used in a "carrot and stick" manner-financial incentives to be vaccinated and financial penalties for not being vaccinated. As stated before, across the U.S. there are laws requiring students to be vaccinated to be enrolled in public schools, and depending on the state, the laws allow religious and/or philosophical exemptions to this policy. In order to make this policy more stringent and prevent abuse of these exemptions, some states have proposed to increase the administrative burden of obtaining an exemption through processing fees and additional reviews of exemption applications [35]. In another study on welfare recipients in Georgia in the 1990s, receiving benefit payments for preschool-age children was tied to proof of up-to-date vaccination status for the children. This monetary sanction was found to increase the proportion of children who achieved vaccine series completion, from $60.6 \%$ to $72.4 \%$ [36]. While evidence shows that these policies can have positive effects on vaccination rates, care must be taken to ensure that these policies do not intensify socioeconomic inequality by having disproportionate burden on minority communities. Notably, the characteristics of undervaccinated children (have received at least one vaccine but are considered not up-to-date) are distinct from those of unvaccinated children (have never received a vaccine), with the former being associated with minority communities, less education, and lower household income, while the latter is associated with more education and higher household income. These results suggest that interventions should be tailored toward individual groups to address the specific causes of vaccine access and/or vaccine hesitancy among those communities [37]. Conversely, monetary incentives may be used to incentivize vaccination and thus elevate vaccination rates. A number of studies have analyzed whether education and outreach or financial incentives are more effective at incentivizing vaccination with varying results, again suggesting that different communities may have different reasons for vaccine hesitancy and interventions should be individualized to achieve maximal effect [38].

Backlash \& institutional distrust: Some potential dangers of mandatory vaccination policies include triggering broad societal backlash, strengthening groups opposing vaccination, and exacerbating existing societal inequality. There are historical examples, for instance, in England and Brazil, where vaccine mandates led to large anti-vaccine movements and riots [39]. Though mandatory vaccination has been shown to enhance vaccination rates in high-income countries such as the U.S., penalties for not adhering to this policy may disproportionately affect minority communities who are distrustful or lack the resources to be vaccinated [40]. This issue is especially visible in the current COVID-19 vaccine rollout. According to an analysis by the Kaiser Family Foundation, by February 1, 2021, Black and Latinx populations have been vaccinated at a significantly lower rate than their respective proportions of the total case count, total death count, and total population [41]. For example, in Delaware, Black and Latinx populations account for only $9 \%$ of vaccinations while accounting for $42 \%$ of cases, $29 \%$ of deaths, and $32 \%$ of the total population. This inequality may be due to a combination of factors, including vaccine access as well as vaccine hesitancy due to concern of adverse effects. Policymakers should weigh these factors heavily and note that mandatory vaccination policies may backfire or result in detrimental societal consequences. Accompanying policies should be crafted to ensure that all communities have an adequate supply of vaccines available to them and additional research should be conducted to devise effective means of encouraging vaccination. Of note, 
vaccine messaging to ease concern about side effects may occasionally have counterintuitive consequences; in a study, researchers showed that only $45 \%$ of parents who received information from the CDC that there is no evidence of a link between the MMR vaccine and autism had intent to vaccinate future children while $70 \%$ of a control group of parents who did not receive this information would vaccinate future children [42], illustrating the delicate balance that must be struck in crafting vaccine messaging.

Success in vaccination uptake depends on a number of factors including the underlying public confidence and trust in medical, public health, and governmental institutions. In the U.S., one's attitude and trust in an institution is shaped by the country's racialized history. Historical oppression of Black, Indigenous, Latinx, and other marginalized groups has manifested in inferior healthcare quality which still persists today [43]. When Black and White adults were asked about their trust in vaccines, both groups distrusted pharmaceutical companies while diverging on trusting federal institutions [44]. White participants implicitly trusted the government, while Black participants distrusted the government and questioned its motives. The distrust among some Black Americans is associated with historical oppression and current care disparities [45]. For example, the "Tuskegee Study of Untreated Syphilis in the Negro", in which Black individuals with syphilis were purposely not given treatment, was conducted by the U.S. Public Health Service and remains an example of unethical medical research [46]. Despite setting human research protection standards in 1979 through the Belmont Report, incidents of unethical medical research on marginalized groups continue to be reported [45]. Institutional skepticism is further reinforced with the performance of the U.S.'s public health system and government response to the COVID-19 crisis, as compared to other governments, in terms of reducing the number of deaths caused by the disease [47]. Some scholars have noted that, "to address distrust, it is critical to recognize that the suspicions, fears, and roots of distrust are logical responses to a history of inequity. It is essential to consider, understand, and address why the suspicion that underlies distrust exists with an individual, practice, organization, or system and match the measurement or intervention strategy to that level" [45]. It remains critical for organizations to be precise and intentional about goals of efforts to promote trust.

Responding to backlash and distrust-communication \& citizen participation: One axis of establishing and maintaining trust that remains important to think through is the communication around vaccines. In Michigan in 2015, public health staff were required to implement vaccine waiver education to those requesting NMEs. These educators initially focused on persuading vaccine-refusing parents through information-based communication, which did little to convince committed vaccine refusers. Instead, the educators switched to cultivating trust through non-adversarial communication techniques such as building rapport and respecting autonomy, finding this to qualitatively be a more effective strategy
[48]. Recently, U.S. participants were surveyed around COVID-19 vaccine messaging and found that effective messaging addresses individuals' concerns, especially those from vulnerable groups including communities of color and low-income individuals, and is truthful and transparent [49]. Participants had the most trust in information coming from healthcare professionals. Thus, thoughtful, culturally relevant messaging coming from health professionals, in addition to community engagement, can be crucial to both informing the community about vaccines and building trust with them.

Advisory panels, task forces, or independent representative bodies can engage local communities in the decision making around vaccination. The COVID-19 pandemic reinvigorated the discussions around human-centered public health interventions, where policy is guided by the complex contexts driven by the diversity of the U.S. population. A "one-size-fits-all approach" of public health solutions does not work for vaccination. Rather, "policymakers need to understand this [societal] diversity and adopt comprehensive local approaches that give communities a voice, and the necessary resources to put ideas into action" [50]. Community-led strategies allow for public ownership and involvement over vaccination programs, help build more robust trust between governance structures and the community, and may allow for more equitable allocation. The 23-person Working Group on Readying Populations for COVID-19 Vaccines conducted a review of what successful general public vaccine uptake would look like and included the significance of community engagement in their conclusions. Their suggestion specifically articulated the need for a national panel convened by an entity like the National Academies of Sciences, Engineering, and Medicine, to create community engagement best practices, and to coordinate with local governments on creating public oversight committees for vaccination programs [51]. Further analysis is needed to precisely quantify the impact of community participation.

\section{Conclusions}

In summary, the benefits to public health that come with increased vaccine uptake are unmistakable; however, a policy of mandatory vaccination may have drawbacks including amplifying existing societal inequality. We have outlined the scientific background of how vaccines are able to prevent infectious disease, described the ethical and legal discussion around mandatory vaccination, presented case studies of vaccination improving public health, and highlighted a handful of key considerations that must be addressed when crafting a mandatory vaccination policy. While mandatory vaccination appears to be the most direct way to improve vaccination rates in the U.S., there may be backlash and distrust that cause this policy to have unintended consequences with disparate effects on certain minority communities. With the goal of reducing infectious disease burden, additional programs beyond the scope of this article must be considered including education to ensure people understand the benefits of vaccination, transparency in vaccine development and approval processes, and equitable distribution and availability of vaccines. 


\section{Acknowledgements}

The authors would like to thank the Science Policy Review leadership team and editorial staff, and the reviewers for their helpful feedback.

\section{Citation}

Cheng, S. S. \& Lepe, B. A. The path to herd immunity: is mandatory vaccination the answer? MIT Science Policy Review 2, 82-89 (2021). https://doi.org/10.38105/ spr. 7 of zvhm6x9.

\section{Open Access}

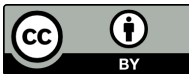

This MIT Science Policy Review article is licensed under a Creative Commons Attribution 4.0 International License, which permits use, sharing, adaptation, distribution and reproduction in any medium or format, as long as you give appropriate credit to the original author(s) and the source, provide a link to the Creative Commons license, and indicate if changes were made. The images or other third party material in this article are included in the article's Creative Commons license, unless indicated otherwise in a credit line to the material. If material is not included in the article's Creative Commons license and your intended use is not permitted by statutory regulation or exceeds the permitted use, you will need to obtain permission directly from the copyright holder. To view a copy of this license, visit http: //creativecommons.org/licenses / $\mathrm{by} / 4.0 /$.

\section{Legislation Cited}

(1) Enforcement of vaccination of inhabitants of towns, MGL c.111 $\S 181$.

(2) U.S. Const. Amend. XIV, § 1.

(3) Jacobson v. Massachusetts, 197 U.S. 11, 25 S. Ct. 358, 49 L. Ed. 643 (1905).

(4) Zucht v. King, 260 U.S. 174, 43 S. Ct. 24, 67 L. Ed. 194 (1922).

(5) National Childhood Vaccine Injury Act, 42 U.S.C. §§ 300aa1-34 (1986).

(6) L.D. 798 (129th Legis. 2019).

(7) H.B. 1638, 362 (66th Legis. 2019).

\section{References}

[1] World Health Organization. Immunization coverage (2021). Online: https://www.who.int/en/news-room/factsheets/detail/immunization-coverage. Accessed: July, 2021.

[2] Iwasaki, A. \& Omer, S. B. Why and how vaccines work. Cell 183, 290-295 (2020). https://doi.org/10.1016/j.cell. 2020.09 .040$.

[3] Kurupati, R. et al. Race-related differences in antibody responses to the inactivated influenza vaccine are linked to distinct pre-vaccination gene expression profiles in blood. Oncotarget 7, 62898-62911 (2016). https://doi.org/10.18632/ oncotarget.11704.

[4] Centers for Disease Control and Prevention. Polio vaccine effectiveness and duration of protection (2018). Online: https://www.cdc.gov/vaccines/vpd/polio/hcp/ effectiveness-duration-protection.html. Accessed: July, 2021.

[5] Centers for Disease Control and Prevention. Vaccines and preventable diseases: measles (2021). Online: https: //www.cdc.gov/vaccines/vpd/measles/index.html. Accessed: July, 2021.
[6] Measles \& Rubella Initiative. Measles \& rubella move fast (2020). Online: https://measlesrubellainitiative.org/ resources/advocacy-tools/infographic/. Accessed: July, 2021.

[7] Polack, F. P. et al. Safety and efficacy of the BNT162b2 mRNA COVID-19 vaccine. New England Journal of Medicine 383, 2603-2615 (2020). https://doi.org/10.1056/ NEJMoa2034577.

[8] Baden, L. R. et al. Efficacy and safety of the mRNA-1273 SARS-CoV-2 vaccine. New England Journal of Medicine 384, 403-416 (2021). https://doi.org/10.1056/ NEJMoa2035389.

[9] Paules, C. I., Sullivan, S. G., Subbarao, K. \& Fauci, A. S. Chasing seasonal influenza-the need for a universal influenza vaccine. New England Journal of Medicine 378, 7-9 (2018). https: //doi.org/10.1056/NEJMp1714916.

[10] Fine, P., Eames, K. \& Heymann, D. L. "Herd immunity": a rough guide. Clinical Infectious Diseases 52, 911-916 (2011). https: //doi.org/10.1093/cid/cir007.

[11] Centers for Disease Control and Prevention. Pandemic planning scenarios (2021). Online: https://www. cdc.gov/coronavirus/2019-ncov/hcp/planningscenarios.html. Accessed: July, 2021.

[12] World Health Organization. Immunizations, vaccines and biologicals: measles (2020). Online: https: / /www. who. int/ immunization/monitoring_surveillance/burden/

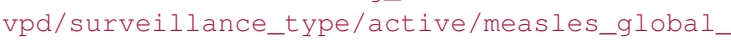
coverage_2019.jpg?ua=1. Accessed: July, 2021.

[13] Wakefield, A. J. et al. RETRACTED: Ileal-lymphoid-nodular hyperplasia, non-specific colitis, and pervasive developmental disorder in children. The Lancet 351, 637-641 (1998). https: //doi.org/10.1016/S0140-6736(97)11096-0.

[14] DeStefano, F., Bodenstab, H. M. \& Offit, P. A. Principal controversies in vaccine safety in the United States. Clinical Infectious Diseases 69, 726-731 (2019). https://doi.org/ $10.1093 / \mathrm{cid} / \mathrm{ciz135.}$

[15] Wombwell, E., Fangman, M. T., Yoder, A. K. \& Spero, D. L. Religious barriers to measles vaccination. Journal of Community Health 40, 597-604 (2015). https://doi.org/10.1007/ s10900-014-9956-1.

[16] Wouters, O. J. et al. Challenges in ensuring global access to COVID-19 vaccines: production, affordability, allocation, and deployment. The Lancet 397, 1023-1034 (2021). https: //doi.org/10.1016/s0140-6736(21)00306-8.

[17] Wadman, M. Public needs to prep for vaccine side effects. Science 370, 1022 (2020). https://doi.org/10.1126/ science.370.6520.1022.

[18] Le, T. T. et al. The COVID-19 vaccine development landscape. Nature Reviews Drug Discovery 19, 305-306 (2020). https: //doi.org/10.1038/d41573-020-00073-5.

[19] Beauchamp, T. L. \& Childress, J. F. Principles of Biomedical Ethics (Oxford University Press, USA, 2013).

[20] Orenstein, W. A. \& Ahmed, R. Simply put: vaccination saves lives 114, 4031-4033 (2017). https://doi.org/10.1073/ pnas.1704507114.

[21] Entwistle, V. A., Carter, S. M., Cribb, A. \& McCaffery, K. Supporting patient autonomy: the importance of clinician-patient relationships. Journal of General Internal Medicine 25, 741-745 (2010). https://doi.org/10.1007/s11606-010-12922.

[22] Rubinstein Reiss, D. Litigating alternative facts: school vaccine mandates in the courts. Journal of Constitutional Law 21, 207 (2018). https://doi.org/10.2139/ssrn.3119970.

[23] National Conference of State Legislatures. States with religious and philosophical exemptions from school immunization requirements (2021). Online: https://www.ncsl.org/ research/health/school-immunization-exemptionstate-laws. aspx. Accessed: July, 2021.

[24] Silverman, R. D., Opel, D. J. \& Omer, S. B. Vaccination over parental objection-should adolescents be allowed to 
consent to receiving vaccines? New England Journal of Medicine 381, 104-106 (2019). https : / / doi . org/10 .1056/ NEJMp1905814.

[25] Gay, N. J. The theory of measles elimination: implications for the design of elimination strategies. The Journal of Infectious Diseases 189, S27-S35 (2004). https://doi.org/10. $1086 / 381592$.

[26] Gastanaduy, P. A. et al. Manual for the surveillance of vaccine-preventable diseases: measles. Tech. Rep. (2019). Online: https://www.cdc.gov/vaccines/pubs/ surv-manual/chpt07-measles.html\#f7. Accessed: July, 2021.

[27] Patel, M. et al. National update on measles cases and outbreaks - United States, January 1-October 1, 2019. Morbidity and Mortality Weekly Report 68, 893-896 (2019). https://doi. org/10.15585/mmwr.mm6840e2.

[28] Barbot, O. Order of the commissioner. Tech. Rep. (2019). Online: https://www1.nyc.gov/assets/doh/downloads/ pdf/press/2019/emergency-orders-measles.pdf. Accessed: July, 2021.

[29] Seither, R. et al. Vaccination coverage with selected vaccines and exemption rates among children in kindergarten - United States, 2018-19 school year. Morbidity and Mortality Weekly Report 68, 905-912 (2019). http://doi.org/10.15585/ mmwr.mm6841e1.

[30] Garnier, R., Nedell, E. R., Omer, S. B. \& Bansal, S. Getting personal: how childhood vaccination policies shape the landscape of vaccine exemptions. Open Forum Infectious Diseases 7, ofaa088 (2020). https://doi.org/10.1093/ ofid/ofaa088.

[31] Zipprich, J. et al. Measles outbreak-California, December 2014-February 2015. Morbidity and Mortality Weekly Report 64, 153-154 (2015). https://www.ncbi.nlm.nih.gov/pmc/ articles/PMC 4584705/.

[32] Navin, M. C. \& Largent, M. A. Improving nonmedical vaccine exemption policies: three case studies. Public Health Ethics 10, 225-234 (2017). https://doi.org/10.1093/phe/phw047.

[33] Rota, J. S. et al. Processes for obtaining nonmedical exemptions to state immunization laws. American Journal of Public Health 91, 645-648 (2001). https: / / doi .org/10.2105/ajph. 91. 4.645 .

[34] Williams, J. T., Rice, J., Cox-Martin, M., Bayliss, E. A. \& O'Leary, S. T. Religious vaccine exemptions in kindergartners: 2011-2018. Pediatrics 144, e20192710 (2019). https : / doi. org/10.1542/peds.2019-2710.

[35] Billington, J. K. \& Omer, S. B. Use of fees to discourage nonmedical exemptions to school immunization laws in US states. American Journal of Public Health 106, 269-270 (2016). https: //doi.org/10.2105/AJPH.2015.302967.

[36] Kerpelman, L. C., Connell, D. B. \& Gunn, W. J. Effect of a monetary sanction on immunization rates of recipients of aid to families with dependent children. Journal of the American Medical Association 284, 53-59 (2000). https://doi.org/ $10.1001 /$ jama.284.1.53.

[37] Smith, P. J., Chu, S. Y. \& Barker, L. E. Children who have received no vaccines: who are they and where do they live? Pediatrics 114, 187-195 (2004). https://doi.org/10. 1542/peds.114.1.187.

[38] Moran, W. P., Nelson, K., Wofford, J. L., Velez, R. \& Case, L. D. Increasing influenza immunization among high-risk patients: education or financial incentive? The American Journal of Medicine 101, 612-620 (1996). https : / doi .org/10.1016/ S $0002-9343$ (96) $00327-0$.

[39] Conis, E. Vaccine nation: America's changing relationship with immunization (University of Chicago Press, 2015).

[40] Omer, S. B. et al. Nonmedical exemptions to school immunization requirements: secular trends and association of state policies with pertussis incidence. Journal of the American Medical Association 296, 1757-1763 (2006). https://doi. org/10.1001/jama.296.14.1757.
[41] Kaiser Family Foundation. Latest data on COVID-19 vaccinations race/ethnicity (2021). Online: https : / Www.kff. org/coronavirus-covid-19/issue-brief/latestdata-on-covid-19-vaccinations-race-ethnicity/. Accessed: July, 2021.

[42] Nyhan, B., Reifler, J., Richey, S. \& Freed, G. L. Effective messages in vaccine promotion: a randomized trial. Pediatrics 133, e835-e842 (2014). https: / / doi .org/10.1542/peds. 2013-2365.

[43] Smedley, B. D., Stith, A. Y. \& Nelson, A. R. Unequal Treatment: Confronting Racial and Ethnic Disparities in Health Care (National Academic Press, US, 2003).

[44] Jamison, A. M., Quinn, S. C. \& Freimuth, V. S. "You don't trust a government vaccine": narratives of institutional trust and influenza vaccination among African American and white adults. Social Science \& Medicine 221, 87-94 (2019). https : / / doi. org/10.1016/j.socscimed.2018.12.020.

[45] Griffith, D. M., Bergner, E. M., Fair, A. S. \& Wilkins, C. H. Using mistrust, distrust, and low trust precisely in medical care and medical research advances health equity. American Journal of Preventive Medicine 60, 442-445 (2021). https: / / doi .org/ $10.1016 / j$.amepre.2020.08.019.

[46] Washington, H. A. Medical apartheid: The dark history of medical experimentation on Black Americans from colonial times to the present (Doubleday Books, 2006).

[47] Devlin, K. \& Kent, N. As pandemic continues, more in US and Europe feel major impact on their lives. Pew Research Center (2021). Online: https://www.pewresearch.org/global/ 2021/02/03/as-pandemic-continues-more-in-u-sand-europe-feel-major-impact-on-their-lives/. Accessed: July, 2021.

[48] Navin, M. C., Kozak, A. T. \& Clark, E. C. The evolution of immunization waiver education in Michigan: a qualitative study of vaccine educators. Vaccine 36, 1751-1756 (2018). https: //doi.org/10.1016/j.vaccine.2018.02.046.

[49] Malik, A. A., McFadden, S. M., Elharake, J. \& Omer, S. B. Determinants of COVID-19 vaccine acceptance in the US. EClinicalMedicine 26, 100495 (2020). https: / / doi . org/10. $1016 / j$.eclinm.2020.100495.

[50] Burgess, R. A. et al. The COVID-19 vaccines rush: participatory community engagement matters more than ever. The Lancet 397, 8-10 (2021). https://doi.org/10.1016/s0140$6736(20) 32642-8$.

[51] Schoch-Spana, M. et al. The public's role in COVID-19 vaccination: human-centered recommendations to enhance pandemic vaccine awareness, access, and acceptance in the United States. Vaccine (2020). https://doi.org/10.1016/ j.vaccine.2020.10.059. 